Página inicial: 29 - Página final: 41

TIPO DE ARTÍCULO: de Investigación

\title{
PUBLINDEX: MÁS QUE UN PROCESO DE INDEXACIÓN ${ }^{4}$.
}

PUBLINDEX: MORE THAN A SIMPLE INDEXING PROCESS.

Recibido: Junio $2014 \quad$ Revisado: Agosto $2014 \quad$ Aceptado: Octubre 2014

Por: Esmeralda Rodriguez ${ }^{1}$, Sandra Naranjo ${ }^{2}$, Dory Luz González. ${ }^{3}$

\section{RESUMEN:}

Este artículo, producto de investigación, expone las principales características del Sistema Nacional de Referencia para la Evaluación de Publicaciones Científicas colombianas (Publindex), logros, avances, interacciones y sus contribuciones a la medición de la publicación científica en el país.

\section{PALABRAS CLAVE:}

revistas científicas, bases de datos, indexación, Publindex.

\begin{abstract}
:
This article, derived from a piece of research, sets out the main features of the National System of Reference for the Evaluation of the Colombian Scientific Publications (Publindex), achievements, progress, interactions, and their contributions to the measurement of scientific publications in the country.
\end{abstract}

\section{KEY WORDS:}

Scientific Journals, Database, Indexing, and Publindex.

\footnotetext{
${ }^{1}$ Profesional en Ciencias de la Información y Documentación. Funcionaria del Departamento Administrativo de Ciencia, Tecnología e Innovación - Colciencias. Bogotá, Colombia. Contacto: contacto@colciencias.gov.co.

${ }^{2}$ Docente-investigadora universitaria Universidad Central, Pedagógica Nacional y Jorge Tadeo Lozano . Licenciada en filosofia y letras, magister en lingüística española; editora y autora de texto educativo en el área de comprensión y producción de textos. Bogotá, Colombia. Contacto: sanenarp@gmail.com.

${ }^{3}$ Docente-investigadora de la Fundación Universitaria Los Libertadores, licenciada en ciencias sociales, especialista en pedagogía, magister en investigación social interdisciplinaria, doctorando es estudios sociales de América Latina. Bogotá, Colombia. Contacto: dlgonzalezh@libertadores.edu.co.
} 


\section{Introducción}

De manera retrospectiva, esto es, mirando los últimos diez a doce años de operación del servicio de indexación de revistas científicas de Departamento Administrativo de Ciencia, Tecnología e Innovación (Colciencias) que recibe el nombre de Publindex, se destaca su naturaleza de instrumento de política pública y sus efectos en términos de la transformación cualitativa del sistema de comunicación formal de la ciencia nacional.

Los criterios de evaluación que Publindex aplica a la clasificación de las revistas han sido apropiados por las comunidades académicas y científicas nacionales, por las editoriales universitarias y por las dependencias responsables de la investigación en las instituciones de educación superior, no solamente de las universidades públicas, sino también de las privadas, lo cual demuestra su nivel de impacto institucional, si se toma en consideración el desarrollo organizacional que se ha generado en torno de los procesos de evaluación de la actividad científica del sistema nacional de ciencia y tecnología.

La evaluación de las publicaciones científicas en el país ha sufrido cambios importantes desde la creación del índice bibliográfico nacional con el objetivo de reunir y evaluar la producción nacional, hasta hoy que la base de datos registra 515 revistas indexadas en las diferentes categorias.

Durantelos años 1995-1998, Colciencias, con el apoyo de investigadores de diferentes áreas del conocimiento, inició un proceso de reconocimiento de las publicaciones colombianas seriadas especializadas de ciencia, tecnología e innovación, para lo cual diseñó y realizó dos convocatorias que permitieron establecer qué instituciones y cómo se estaba realizando la producción de las revistas en el país. En este ámbito se estableció una base nacional que se encargó de medir los niveles de calidad científica, calidad editorial, accesibilidad y visibilidad para luego constituir el Índice Bibliográfico Nacional Publindex, IBN - Publindex.

\section{Metodologia.}

Para realizar el presente artículo, derivado de la investigación "Producción textual para la gestión y divulgación del conocimiento" que se desarrolla en el Departamento de Formación Humana y Social de la Fundación Universitaria de los Libertadores, registrado en la convocatoria de investigación 2014 I+D+I, se tomaron los datos de los últimos diez a doce años de operación del servicio de indexación de revistas científicas de Departamento Administrativo de Ciencia, Tecnología e Innovación (Colciencias) que recibe el nombre de Publindex, realizando:

- Un rastreo documental, archivos de la institución Publindex,

- Un análisis de las bases de datos de la institución Publindex,

- Un análisis comparativo relacional, con otras bases bibliográficas que miden el impacto de las revistas científicas y en las que Colombia, ha logrado genera cierto incremento en su visibilidad.

En dicho rastreo documental, de sus registros y sus bases de datos, se observó:

- Su naturaleza de instrumento de política pública y sus efectos en términos de la transformación cualitativa del sistema de comunicación formal de la ciencia nacional 
- Los indicadores que ha arrojado sobre las revistas que se han acogido a los servicios de indexación que presta,

- La manera como ha dado respuesta a la necesidad de identificar, evaluar y categorizar las revistas nacionales de ciencia, tecnología e innovación, según evidencias de calidad científica, calidad editorial, visibilidad y accesibilidad;

Vale aclarar que dichas categorias se han convertido así mismo, en referencias para calificar la producción de artículos y de diferentes tipos de documentos de investigadores individuales asociados a instituciones nacionales.

\section{Resultados.}

\section{$\underline{\text { La evolución de Publindex }}$}

En el año 1998 se realizó el primer ejercicio de indexación de las publicaciones científicas en el país; en este año ingresaron al índice revistas en las áreas de ciencias biológicas, ciencias básicas y ciencias sociales y humanas.

En 2001 se estableció el primer conjunto de condiciones y requisitos, que luego se ajustó cuando, en junio de 2002, se publicó el Decreto 1279, el cual designó a Colciencias como la entidad nacional responsable de indexar y homologar las publicaciones especializadas de actividades de ciencia, tecnología e innovación (ACTI), a partir de la asignación de cuatro categorias de menor a mayor: C, B, A2 y A1. Este derrotero generó el diseñó del Servicio Nacional de Indexación de Publicaciones Especializadas Seriadas de Ciencia, Tecnología e Innovación - Publindex, que se puso a disposición de la comunidad científica nacional en diciembre de 2002, con la función de determinar la calidad de las publicaciones científicas, proceso conocido como indexación, y que se realiza según criterios de calidad cientifica y editorial, estabilidad y visibilidad.

Para cumplir con esta función, se creó el modelo de análisis y se diseñó un repositorio digital que recoge el histórico documental de las revistas de forma permanente, de modo que recolecta y actualiza la información de las publicaciones nacionales que voluntariamente solicitan hacer parte de la Base Bibliográfica Nacional - BBN Publindex. La información recolectada se constituye en una fuente de información sobre los resultados de investigación, que circulan en las revistas nacionales, lo cual contribuye a la construcción de representaciohbnes de las dinámicas de la producción científica nacional y fomenta la elaboración de políticas por parte de los mismos investigadores, de las instituciones o del Sistema Nacional de Ciencia y Tecnología e Innovación (SNCTI). Los datos que analiza Publindex en las publicaciones científicas permiten realizar representaciones de los niveles de calidad y desarrollo de dichas publicaciones.

Entre el año 2001 a 2013 ingresaron a la Base Bibliográfica Nacional - BBN Publindex 830 títulos que fueron analizados y clasificados, y 515 de estos entraron a conformarlo. Actualmente, de acuerdo con las áreas de conocimiento que propone la Organización para la Cooperación y el Desarrollo Económico (OCDE), hay publicaciones indexadas en las áreas de: ciencias agrícolas, ciencias médicas y de la salud, ciencias naturales y exactas, ciencias sociales, humanidades, ingeniería y tecnología (ver tabla 1). También hay un grupo de revistas multidisciplinarias que, con el tiempo, deberán especializarse en un área de conocimiento, si quieren 
continuar siendo visibles a nivel internacional en los sistemas de indexación y resumen (SIR).

Tabla 1. Número de revistas por áreas de conocimiento OCDE

\begin{tabular}{|l|c|c|c|c|r|}
\hline & $\begin{array}{c}\text { Cat. } \\
\text { AREAS OCDE }\end{array}$ & $\begin{array}{c}\text { Cat. } \\
\text { A2 }\end{array}$ & & & \\
\hline Ciencias agricolas & 2 & 8 & 7 & 7 & $\mathbf{2 4}$ \\
\hline Ciencias médicas y de la salud & 6 & 25 & 18 & 34 & $\mathbf{8 3}$ \\
\hline Ciencias naturales y exactas & 4 & 13 & 11 & 16 & $\mathbf{4 4}$ \\
\hline Ciencias sociales & 9 & 40 & 50 & 111 & $\mathbf{2 1 0}$ \\
\hline Humanidades & 1 & 18 & 22 & 27 & $\mathbf{6 8}$ \\
\hline Ingeniería y tecnologia & 4 & 10 & 9 & 31 & $\mathbf{5 4}$ \\
\hline Multidisciplinarias & 0 & 5 & 3 & 24 & $\mathbf{3 2}$ \\
\hline Total & 26 & 119 & 120 & 250 & $\mathbf{5 1 5}$ \\
\hline
\end{tabular}

Fuente: elaboración propia con base en datos del Índice Bibliográfico Nacional - Publindex, 2013

\section{Tipologia de los sistemas de indexación y resumen}

Los sistemas de indexación y resumen son bases de datos que diversas instituciones de orden nacional e internacional utilizan para evaluar y clasificar revistas especializadas en la divulgación de ACTI. Estos se clasifican, de acuerdo con Colciencias, en los siguientes tipos:

Índices bibliográficos de citaciones (IBC): Con base en análisis estadísticos de las citas que reciben las revistas, calculan el llamado factor de impacto y establecen con base en él su ordenamiento. Índices bibliográficos (IB): Seleccionan revistas científicas utilizando estrictas exigencias científicas y editoriales. Tienen comités que se encargan, de una parte, del análisis calcográfico de la revista, esto es el comité editorial; y de otra, de la evaluación de la calidad científica, la originalidad y la pertinencia de los documentos publicados, esto es el comité científico. Estos IB son construidos por asociaciones cientificas, universidades, instituciones académicas, institutos especializados en el análisis de la información científica o agencias que apoyan a la actividad científica. Bases Bibliográficas con Comité de Selección (BBCS): A diferencia de los índices bibliográficos, centran su objeto en la selección de artículos de investigación publicados en revistas arbitradas; poseen un comité de selección, encargado de verificar la pertinencia con respecto al interés temático de la base y la calidad de los artículos, así como el cumplimiento de las normas editoriales internacionales exigidas para la publicación de artículos cientificos. Estas BBCS son construidas o avaladas por asociaciones científicas, universidades, instituciones académicas o institutos especializados en el análisis de la información científica (Departamento Administrativo de Ciencia, Tecnología e Innovación - Colciencias., 2013).

Para realizar la inclusión en estas bases de datos, tanto Publindex, como las demás bases de datos, realizan el proceso de clasificación de las revistas basándose en la información integrada permanentemente por parte de los editores. A partir de esta información, los sistemas de indexación recogen las actualizaciones que las revistas hacen en los periodos determinados por estos y con los resultados se actualizan los SIR, incluido el IBN - Publindex, lo cual significa que cada revista va 
construyendo su propio histórico dentro se conserva tanto la información general como sus contenidos, las sucesivas indexaciones y las categorias en que han sido clasificadas, así como los períodos de su vigencia.

\section{$\underline{\text { La evaluación de Publindex en cifras }}$}

Como sistema nacional de indexación de revistas científicas, Publindex ha generado indicadores que permiten la evaluación de publicaciones científicas colombianas en ACTI. Estos indicadores miden la calidad científica, la calidad editorial, la visibilidad y la accesibilidad de las revistas. También ha establecido la definición del artículo cientifico y ha propuesto tipologias que se usan para calificar la calidad de artículo de divulgación científica de la producción textual de los investigadores asociados a instituciones nacionales

Desde su creación, Publindex se ha convertido en una herramienta clave para la operatividad del Decreto 1279 de 2002, en el que define el régimen salarial y prestacional de los docentes vinculados a las universidades estatales, en términos del cumplimiento del Artículo 10 que se refiere al reconocimiento de los puntajes de la productividad académica relacionada con las ACTI que es divulgada en revistas científicas registradas en el IBN; también la universidades privadas han generado sus propios modelos de reconocimiento con base en este. Sin duda alguna esta alianza político-técnica es uno de los factores responsables del incremento en la productividad y calidad de publicaciones cientificas del país, pero, y más importante aún, produjo una transformación cultural en la apreciación y valoración de la producción científica nacional.

Es importante mencionar que Colciencias, y de acuerdo con el Decreto 1279 de 2002, solo mide las publicaciones en distintos formatos relacionadas con las ACTI, bien sea a través de Publindex (revistas) o bien sea través de otros sistemas, por ejemplo, el reconocimiento de libros resultados de estas actividades que son editado por editoriales reconocidas y calificadas por esta entidad. Existen otras publicaciones colombianas editadas por las instituciones que son no objeto de evaluación o reconocimiento por Colciencias, puesto que no están cubiertas por las ordenanzas del Decreto. Estas publicaciones son objeto de otras gestiones y practicas editoriales de proyección de calidad, como parte de los fines misionales de las instituciones que las producen. 
En las publicaciones reconocidas y calificadas por Publindex, el crecimiento ha sido constante, como puede observarse en la gráfica 1.

Gráfica 1. Aumento de las revistas indexadas, 2002-2013

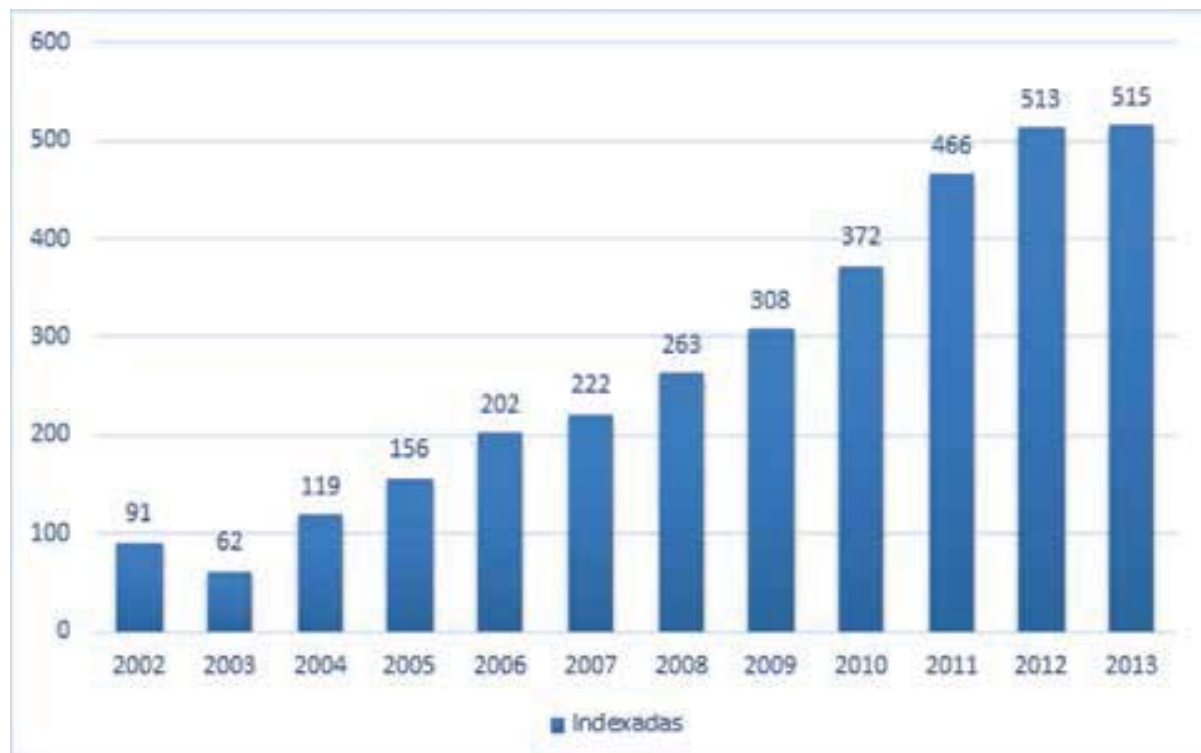

Fuente: datos del Índice Bibliográfico Nacional - Publindex, 2013.

Esta gráfica representa el número de revistas indexadas durante el periodo 20022013. Los dos primeros años presentan los más bajos índices de indexación, dado que corresponde al ciclo en el que los editores se estaban ajustando a las condiciones y los requisitos de Publindex para emprender la labor de analizar sus publicaciones y ajustarse a las directrices; en este sentido, este periodo se considerar el ciclo de aprendizaje en los procesos de calidad cientifica y editorial. A partir del año 2005, las publicaciones mejoran sus procesos editoriales y aseguran la calidad científica de sus artículos, lo que hace que se incremente el número de revistas indexadas, no solo en número sino también en la calidad del proceso (ver tabla 1). En cada categoria: C, B, A1 y A2, el índice va aumentado su presencia, y resulta muy significativo que la categoría $A 1$, la categoría más alta, sea la de mayor crecimiento anual (ver tabla 2). (Departamento Administrativo de ciencia, tecnología e innovacion. Colciencias, 2014b)

Tabla 2. Revistas indexadas por categoria, 2002-2013

\begin{tabular}{|c|c|c|c|c|c|c|c|c|c|c|c|c|}
\hline & & & & & & & & & & & & \\
\hline A1 & 1 & 1 & 1 & 2 & 3 & 5 & 14 & 20 & 23 & 25 & 27 & 26 \\
\hline A2 & 9 & 8 & 9 & 5 & 15 & 31 & 39 & 49 & 72 & 93 & 113 & 126 \\
\hline B & 7 & 3 & 11 & 38 & 44 & 50 & 69 & 74 & 66 & 98 & 110 & 130 \\
\hline C & 74 & 50 & 98 & 111 & 140 & 136 & 141 & 165 & 211 & 250 & 263 & 233 \\
\hline Total & 91 & 62 & 119 & 156 & 202 & 222 & 263 & 308 & 372 & 466 & 513 & 515 \\
\hline
\end{tabular}

Fuente: elaboración propia con base en datos del Índice Bibliográfico Nacional -Publindex 2013. 
El incremento registrado en esta categoría tiene también impacto en los SIR internacionales, lo que ha permitido que la publicación cientifica del país adquiera visibilidad en los ámbitos regional y mundial (ver gráfica 2). (Departamento Administrativo de ciencia, tecnología e innovacion. Colciencias, 2014b)

Gráfica 2. Revistas nacionales registradas en Publindex e indizadas por SIR, según tipo 2002-2009

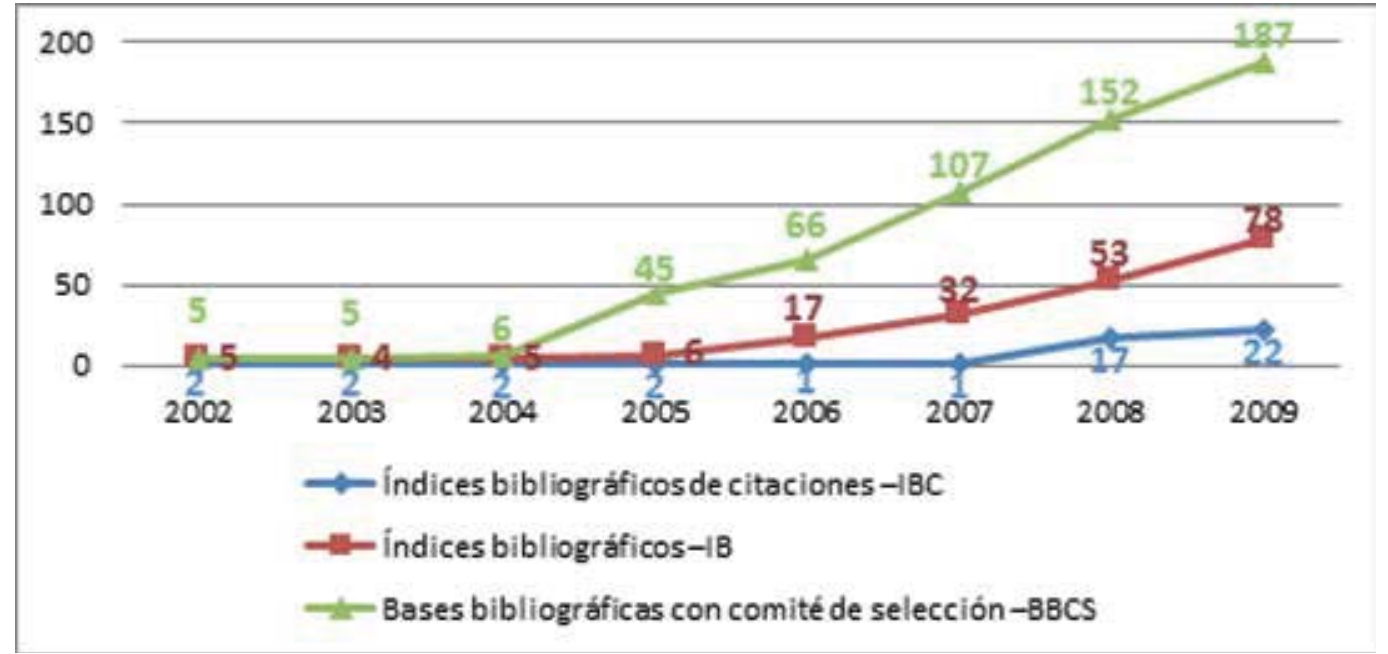

Fuente: datos del Índice Bibliográfico Nacional -Publindex 2013.

En esta gráfica se evidencia el incremento en la visibilidad internacional de las revistas colombianas indexadas en Publindex. A partir de 2005 hay un incremento en la visibilidad por su inclusión en índices bibliográficos -IB como SciELO, Medline y PsycINFO, así mismo por su inclusión en BBCS en diversas áreas del conocimiento. En el caso de los índices bibliográficos de Citación -IBC ha aumentado la inclusión de las revistas nacionales, lo que ha permitido un mayor número de revistas indexadas en categoria A1.

Es pertinente mencionar, sin embargo, que las revistas colombianas han ganado tienen visibilidad en algunos índices bibliográficos citacionales (ver tabla 3), mas no en los de mayor impacto como el Journal Citation Report - JCR de Thomson Reuters o Scimago Journal Ranking -SJR de Scimago Lab, situación que es, actualmente, objeto de estudio de Colciencias para proponer estrategias en el acompañamiento a los editores y a las instituciones. 
PUBLINDEX: MORE THAN A SIMPLE INDEXING PROCESS.

Tabla 3. Número de revistas colombianas indexadas en Publindex cubiertas por SIR internacionales, 2002 - 2013

\begin{tabular}{|c|c|c|c|c|c|c|c|c|c|c|c|c|c|}
\hline SIR & 2001 & 2002 & 2003 & 2004 & 2005 & 2006 & 2007 & 2008 & 2009 & 2010 & 2011 & 2012 & 2013 \\
\hline SciELO & 0 & 0 & 0 & 0 & 1 & 12 & 27 & 46 & 72 & 99 & 112 & 113 & 148 \\
\hline Lilacs & 2 & 0 & 0 & 0 & 12 & 12 & 23 & 39 & 45 & 46 & 58 & 68 & 66 \\
\hline Fuente Académica & 0 & 0 & 0 & 0 & 0 & 0 & 11 & 24 & 37 & 59 & 84 & 88 & 150 \\
\hline IBSS & 0 & 0 & 0 & 0 & 5 & 7 & 12 & 14 & 16 & 17 & 17 & 19 & 19 \\
\hline $\begin{array}{l}\text { Sociological } \\
\text { Abstracts }\end{array}$ & 0 & 0 & 0 & 0 & 2 & 4 & 7 & 9 & 15 & 16 & 11 & 4 & 10 \\
\hline $\begin{array}{c}\text { Commonwealth } \\
\text { Agriculture Bureau }\end{array}$ & 0 & 0 & 0 & 1 & 4 & 5 & 7 & 10 & 14 & 13 & 13 & 10 & 20 \\
\hline $\begin{array}{l}\text { Social Science } \\
\text { Citation }\end{array}$ & 1 & 1 & 1 & 1 & 1 & 1 & 1 & 1 & 3 & 6 & 7 & 7 & 5 \\
\hline EcoLIT & 2 & 4 & 4 & 4 & 4 & 6 & 5 & 7 & 10 & 10 & 10 & 14 & 21 \\
\hline $\begin{array}{l}\text { Science Citation } \\
\text { Index (SCI) }\end{array}$ & 0 & 0 & 0 & 0 & 0 & 0 & 1 & 1 & 6 & 15 & 12 & 12 & 13 \\
\hline $\begin{array}{c}\text { Educational } \\
\text { Research Abstracts } \\
\text { (ERA) }\end{array}$ & 0 & 0 & 0 & 0 & 1 & 4 & 6 & 7 & 7 & 9 & 9 & 9 & 15 \\
\hline Georef & 0 & 0 & 0 & 0 & 1 & 5 & 4 & 4 & 6 & 6 & 8 & 6 & 5 \\
\hline PsycINFO & 1 & 2 & 2 & 2 & 3 & 3 & 3 & 5 & 5 & 5 & 5 & 5 & 8 \\
\hline Biological Abstracts & 0 & 0 & 0 & 0 & 3 & 4 & 3 & 4 & 5 & 5 & 5 & 5 & 3 \\
\hline Philosopher index & 0 & 0 & 0 & 0 & 2 & 3 & 5 & 6 & 5 & 6 & 5 & 6 & 8 \\
\hline Chemical abstracts & 0 & 0 & 0 & 0 & 3 & 1 & 4 & 5 & 5 & 5 & 2 & 5 & 11 \\
\hline Zoo-rec & 0 & 0 & 0 & 0 & 2 & 3 & 4 & 4 & 4 & 3 & 5 & 4 & 6 \\
\hline $\begin{array}{c}\text { Linguistics and } \\
\text { language behavior } \\
\text { abstracts }\end{array}$ & 1 & 0 & 0 & 0 & 1 & 1 & 2 & 4 & 4 & 4 & 4 & 6 & 6 \\
\hline Biosis & 0 & 0 & 0 & 0 & 3 & 3 & 2 & 2 & 3 & 2 & 5 & 5 & 7 \\
\hline Asfa & 0 & 0 & 0 & 0 & 0 & 2 & 4 & 4 & 3 & 3 & 3 & 1 & 5 \\
\hline Math-R & 0 & 0 & 0 & 0 & 1 & 2 & 2 & 2 & 3 & 2 & 3 & 2 & 3 \\
\hline Inspec & 0 & 0 & 0 & 0 & 0 & 1 & 1 & 3 & 3 & 5 & 3 & 4 & 6 \\
\hline Zentralblatt MATH & 0 & 0 & 0 & 0 & 1 & 1 & 4 & 3 & 1 & 2 & 2 & 1 & 1 \\
\hline Index medicus & 0 & 0 & 0 & 0 & 2 & 2 & 1 & 2 & 1 & 1 & 1 & 1 & 1 \\
\hline Curr-ind-stat & 0 & 0 & 0 & 0 & 0 & 1 & 1 & 1 & 1 & 1 & 1 & 1 & 1 \\
\hline $\begin{array}{c}\text { International } \\
\text { Pharmaceutical } \\
\text { Abstracts }\end{array}$ & 0 & 0 & 0 & 0 & 0 & 0 & 0 & 0 & 0 & 0 & 0 & 0 & 0 \\
\hline Metadex & 0 & 0 & 0 & 0 & 0 & 0 & 0 & 0 & 0 & 0 & 0 & 1 & 2 \\
\hline
\end{tabular}

Fuente: elaboración propia con base en datos del Índice Bibliográfico Nacional - Publindex, 2013.

Con base en esta tabla se puede determinar que, a partir del año 2008, se ha visto un incremento o ingreso de las revistas colombianas en sistemas de SIR internacionales, y algunas se encuentran indexadas en varios SIR, razón por la cual los totales sobrepasan los 26 los SIR reconocidos por el proceso, en los cuales una revista puede estar indexada en más de uno. También se hace claro que es necesaria la actualización de los SIR en áreas del conocimiento como las ciencias sociales, la ingeniería y las humanidades.

$\underline{\text { Los principales Logros de Publindex }}$

La indexación de las revistas nacionales ha permitido estandarizar las publicaciones de los resultados de las ACTI, promocionar la evaluación por pares como práctica institucionalizada y aceptada, aumentar la cualificación de la producción científica nacional publicada en las revistas, incrementar la internacionalización de los medios de circulación, promover la exogamia y la visibilidad nacional e internacional de la publicación científica, a través de la integración de las revistas SIR; establecer el 
grado de desarrollo de las revistas científicas, y obtener información valiosa para la toma de decisiones por parte de las instituciones educativas y sus diferentes estamentos, respecto a sus publicaciones.

Progresivamente, la indexación en Publindex les ha permitido a la revistas construir características específicas, integrar normas, definir su propia audiencia, asegurar su estabilidad, y ganar reconocimiento y visibilidad en los grupos sociales a los que pretende satisfacer con el saber certificado.

Además de lo anterior, Publindex, construye y representa las dinámicas nacionales de la investigación que dejan registro en las publicaciones especializadas y en la Base; promueve la visibilidad y reconocimiento de la producción científica nacional a través de la articulación con otros sistemas de información, entre los más destacados, Scientific Electronic Library Online (SciELO-Brasil), Latindex (México) y Gale Learning (base de datos de México).

Igualmente, este sistema de indexación promueve la apropiación social del conocimiento, a través de su difusión en medios de comunicación y formación de mediadores de ACTI, así como con el apoyo a entidades que cumplan esta labor. También ha servido para mejorar los procedimientos de edición y publicación de las revistas editadas por instituciones universitarias privadas y públicas, y ha fomentado la coordinación de publicaciones en algunas universidades para centralizar las políticas o líneas editoriales, no solo relacionadas con el proceso de indexación en Publindex, sino también dentro de la misma institución con otros sistemas internacionales y con sellos editoriales que se han venido formando en estas instituciones.

\section{Conclusiones.}

En los procesos de acreditación institucional y aseguramiento de la calidad que vienen desarrollando las más importantes universidades del país, Publindex apoya, fundamentalmente, la acreditación de calidad de los programas académicos de pregrado y posgrado, y la selección de profesores e investigadores.

En el primero, cabe mencionar que, desde el año 2006, cuando el Ministerio de Educación Nacional (MEN) anunció la entrada en vigencia del sistema de acreditación de calidad de la educación superior, Publindex juega un importante papel para las instituciones en el área institucional y en el área de la oferta académica (Consejo Nacional de Acreditación, 2010).

En el área institucional, el MEN, a través del CNA, propone fomentar la pertinencia y impacto social de la educación superior, mediante la difusión de conocimiento en medios académicamente reconocidos y la publicación de la producción científica, técnica, artística, humanística y pedagógica (Consejo Nacional de Acreditación, 2010)0, lo cual tiene un indicador de existencia y medición en Publindex.

En el área de la evaluación de la calidad académica, que mide la producción científica de profesores y alumnos, así como la calidad, pertinencia y producción científica de los programas de pregrado y posgrado (Consejo Nacional de Acreditación, 2010), Publindex contribuye a hacer evidentes los logros de los profesores que hacen parte de los programas académicos de las instituciones, puesto que el proceso de indexación está directamente articulado con la medición de grupos de investigación 
en ciencia, tecnología e innovación. Los resultados del proceso de medición de grupos son importantes para las instituciones porque, con base en estos, se pueden tomar decisiones con relación a reformas curriculares, al fortalecimiento de ofertas en maestrías y doctorados, a la formulación de planes de desarrollo y a la institucionalismo, capacitación y formación de docentes, entre otros.

Por otra parte, la publicación de los resultados de la investigación y el impacto de esta, es uno de los factores más relevante para el MEN a la hora de evaluar la calidad de los programas académicos, especialmente los de posgrado (Consejo Nacional de Acreditación, 2010). Para el primer caso, se considera la cantidad de publicaciones científicas en los últimos cinco años en revistas nacionales indexadas en Publindex, y para el segundo, el número de artículos de investigación generados por personas vinculadas al programa en los últimos cinco años (Consejo Nacional de Acreditación, 2010).

En relación con las exigencias del MEN, es evidente que Publindex juega un papel fundamental en los procesos de acreditación de alta calidad de las instituciones de educación superior y de sus programas académicos; sin embargo es necesario sentar bases para reflexionar sobre las condiciones actuales de Publindex para esta participación, puesto que hasta el 2013 este servicio nacional de indexación apoyaba plenamente la medición y clasificación de grupos de investigación de las instituciones, en la medida en la que los artículos en las revistas indexadas en Publindex eran tenidos en cuenta como "productos de nuevo conocimiento", y de acuerdo con las categorias de las revista se les asignaba pesos en la medición: en revistas A, peso 1; en $\mathrm{B}, 0,7$; y en $\mathrm{C}, 0,4$; pero, a partir de la convocatoria 640 de 2013 para el reconocimiento y medición de grupos de investigación, desarrollo tecnológico y/o innovación, así como para el reconocimiento de investigadores, su papel ya no fue tan representativo.

Las condiciones establecidas en dicha convocatoria implicaron el denominado factor de impacto, esto es, el reconocimiento de las citaciones, índice de citación (IC), que recibe un artículo científico en otros de la misma índole, y solo algunos de los SIR miden este factor. La forma de determinar el factor de impacto en estos SIR consiste en dividir el número de citaciones de una revista al año por la cantidad de artículos que se publicaron en ese periodo y categorizar con base una categoria denominada cuartil, que separa las revistas de acuerdo con las áreas de conocimiento y las ordenar de mayor a menor en cuatro partes iguales, de tal forma que cada parte es un cuartil; las revistas con mayor IC se ubican en el primer cuartil, y van descendiendo, en relación con tal índice, al segundo, al tercero y al cuarto, que es el más bajo.

De acuerdo con las nuevas condiciones, "en todos los articulos publicados en revistas indexadas en los indices bibliográficos de citaciones (ISI o Scopus), la categoria se asignará de acuerdo con el cuartil que ocupe la revista en dichos sistemas de indices de citación (correspondiendo el cuartil superior Q1 al tipo A1, el Q2 al tipo A2, el Q3 al tipo B y el Q4 al tipo C)" (Departamento Administrativo de ciencia, tecnología e innovacion. Colciencias, 2014b), y estos son los que tienen más peso en la medición de los artículos científicos registrados dentro de la producción de un grupo de investigación. En la reforma, se sigue reconociendo las contribuciones en publicaciones que no están incluidos en dichos SIR, pero con puntajes y pesos más bajos. 
El Índice Bibliográfico Nacional-IBN, Publindex, es una de las herramientas más importantes en sistema nacional que mide los resultados de las actividades de ciencia, tecnología e innovación en el país, el SCIENTI.

Su papel ha sido fundamental en el aumento de las publicaciones científicas y de la calidad de estas, pero es necesario reflexionar sobre sus actuales condiciones para fortalecer sus relaciones con los demás componentes del sistema, como el sistema de medición de los grupos de investigación.

Actualmente, la política de indexación está siendo ajustada con el fin de mejorar dicha relación, así como para incrementar y fortalecer la calidad, cantidad, visibilidad, consumo, uso y reconocimiento de los artículos científicos publicados en revistas académicas y científicas nacionales, para lo cual se ha establecido una estrategia tendiente a revisar las definiciones de las tipologias de los artículos y la clasificación de los mismos, construir y medir indicadores que generen en las publicaciones científicas nacionales el incremento cuantitativo como cualitativo de la producción, y aumentar la lista de sistemas de indexación y resumen -SIR, que se basa en modificar los parámetros de medición de visibilidad.

Dentro de esta estrategia, también se busca articular el componente del sistema nacional de información de ciencia, tecnologia e innovación con otros sistemas de información de países de la región; promover e impulsar la creación de redes colaborativas entre el sector privado, público y la academia para desarrollar revistas con altos estándares de calidad cientifica y con proyección internacional; implementar TIC en la administración y producción de revistas especializadas de ACTI, y promover la cultura Open Access entre las revistas científicas nacionales y su inclusión en bases de datos de acceso abierto y en repositorios. 


\section{Referencias Biliográficas.}

Consejo Nacional de Acreditación. (Mayo de 2010). Acreditacion de Alta Calidad Posgrados. Recuperado el 24 de Febero de 2014, de cna.gov.co: http://www. cna.gov.co/1741/article-186363.html

Departamento Administrativo de Ciencia, Tecnologia e Innovación - Colciencias. (Julio de 2013). Servicios de indexación y resumen - SIR utilizados para los procesos de indexación y homologación de Revistas Especializadas de CTI. Recuperado el 2 de Febrero de 2014, de Colciencias: http://www.colciencias. gov.co/sites/default/files/ckeditor_files / files / Documento\%20SIRES\%20-\%20 2013.pdf

Departamento Administrativo de ciencia, tecnología e innovación. Colciencias. (Julio de 2008). Modelo de medeción de grupos de investigaicon, tecnológica o de innovacion. Recuperado el 1 de Junio de 2014, de usergioarboleda.edu. co: http:/ / www.usergioarboleda.edu.co/civilizar/documentos/instructivos_ formatos/modelo_medicion_grupos_2008_colciencias.pdf

Departamento Administrativo de ciencia, tecnología e innovacion. Colciencias. (Marzo de 2014b). Indicadores Pulbindex 2002-2011. Recuperado el 20 de Mayo de 2014, de ocyt.org.co: http://ocyt.org.co/informes_indicadores/Indicadores_ OCyT_2012/html/files/assets/basic-html/page72.html

Departamento Administrativo de Ciencia, Tecnología e Innovación. Colciencias. (Julio de 2013). Sistema Nacional de Ciencia, Tecnología e innovación. Colciencias. Recuperado el 10 de Mayo de 2014, de Colciencias: http://www.colciencias.gov. co/programas_estrategias

Departamento Administrativo de ciencia, teconología e innovación. Colciencias. (15 de Octubre de 2014c). Modelo de medición de grupos de investigacion, desarrollo tenológico o de innovación y de reconocimiento de investigadores del sistema nacional de cienc ias y tecnologia. Recuperado el 15 de Noviembre de 2014, de Colciencias: http://www.colciencias.gov.co/sites/default/files/ ckeditor_files /files / DOCUMENTO\%20MEDICI\%C3\%93N\%20GRUPOS\%20-\%20 INVESTIGADORES\%20VERSI\%C3\%93N\%20FINAL\%2015\%2010\%202014\%20 (1)(1).pdf

Departamento Nacional de Planeación . (27 de Abril de 2009). Conpes 3582: Política Nacional de Ciencia, Tecnología e innovacion. Recuperado el 2 de Noviembre de 2014, de autonoma.edu.co: http://www.autonoma.edu.co/media/documentosinvestigacion/02-conpes-3582-CyT.pdf

Ley 1286, Nueva Ley de ciencia, tecnología e innovación (Congreso de la República de Colombia Enero de 2009). 


\section{Notas}

${ }^{4}$ Este artículo es producto del proyecto de investigación "Producción textual para la gestión y divulgación del conocimiento" que se desarrolla en el Departamento de Formación Humana y Social de la Fundación Universitaria de los Libertadores, registrado en la convocatoria de investigación $2014 \mathrm{I}+\mathrm{D}+\mathrm{I}$. 